\title{
ELECTRON COOLING IN STORAGE RINGS
}

\author{
J. S. BELL and M. BELL \\ CERN, Geneva, Switzerland \\ (Received February 2, 1981)
}

\begin{abstract}
It is found that the effect of "flattening" of the electron velocity distribution is to increase the rate of cooling of small betatron oscillations by a factor of 2.4 , and not by a factor of 4 as often quoted. This is when the cooler magnetic field is ignored. When it is allowed for, in the usual way, the cooling rate involves a divergent integral whose regulation depends on the details of particular cases.
\end{abstract}

\section{to Y. ORLOV}

\section{INTRODUCTION}

This is a revision of some aspects of the theory of electron cooling ${ }^{1}$ of ion beams in storage rings. We consider a Maxwellian distribution of electron velocities, and the corresponding "flattened" distribution in which longitudinal velocity spread is suppressed. While agreeing with results given in the literature for the Maxwellian case, we find that "flattening" increases the rate of damping of small betatron oscillations only be a factor of 2.4, and not by a factor of 4 as often quoted. This is when the cooler magnetic field is ignored. When it is allowed for, in the usual way, we find for the "flattened" case a divergent integral in the cooling rate of idealized betatron oscillations. It arises because the damping force increases as transverse velocity decreases, and arbitrarily small velocities occur in the course of oscillation. The regulation of this integral depends on how the idealizations and approximations of the theory fail in particular cases.

Let the electron velocity distribution be

$$
f\left(\mathbf{V}_{e}\right) d^{3} \mathbf{V}_{e}
$$

with

$$
\int d^{3} \mathbf{V}_{e} f\left(\mathbf{V}_{e}\right)=1 .
$$

The Maxwellian distribution, in the rest frame of the electron gas, is

$$
f\left(\mathbf{V}_{e}\right)=\left(2 \pi k T / m_{e}\right)^{-3 / 2} \exp \left(-\frac{1}{2} m_{e} \mathbf{V}_{e}^{2} / k T\right),
$$

where $m_{e}$ is electron mass and $T$ temperature and $k$ is Boltzmann's constant. The corresponding flattened distribution is

$$
\begin{aligned}
f\left(\mathbf{V}_{\mathcal{e}}\right)= & \left.(2 \pi k T) / m_{e}\right)^{-1} \delta\left(V_{e x}\right) \\
& \times \exp \left[-\frac{1}{2} m_{e} \mathbf{V}_{\mathcal{C}_{\perp}}^{2} /(k T)\right],
\end{aligned}
$$

where

$$
\mathbf{V}_{e^{+}}^{2}=V_{e^{2}}^{2}+V_{e^{\prime} y}^{2}
$$

Note that in both cases mean square transverse velocity components are given by

$$
\begin{aligned}
& \left\langle V_{e y}^{2}\right\rangle=\left\langle V_{e z}^{2}\right\rangle=k T / m_{e} \\
& \left\langle V_{e^{\perp}}^{2}\right\rangle=2 k T / m_{e} .
\end{aligned}
$$

Betatron oscillation cooling times are often written as ${ }^{1-3}$

$$
\tau=\kappa\left(L_{c} \cdot r_{p} r_{e} n_{e} c\right)^{-1}\left(m_{e} c^{2} / k T\right)^{-3 / 2} \gamma^{2} / d,
$$

where $\kappa$ is a numerical constant, $L_{e}$ is the "Coulomb logarithm", $r_{e}$ is the "classical electron radius" $=2.82 \times 10^{-13} \mathrm{~cm}, r_{i}$ is the "classical ion radius" $=Z_{i} r_{e} m_{e} / m_{i}, m_{i}$ is ion mass, $Z_{i}$ is ion charge number, $n_{e}$ is electron density, in lab frame, $c$ is velocity of light, $\gamma=\left(1-\beta^{2}\right)^{-1 / 2}$, $\beta=V_{0} / c, V_{0}$ is electron beam velocity, and $d$ $=($ cooler length $) /($ orbit length $)$.

The reading of the literature is complicated by the fact that Ref. 2 contains some obvious misprints at the critical point, and (we think) a less obvious one. Presumably following Ref. 2, Sørensen ${ }^{3}$ quotes formula (8) with the substitution

$$
k T /\left(m_{e} c^{2}\right) \rightarrow\left(\beta \gamma \theta_{c}\right)^{2}
$$


and states that $\beta \gamma \theta_{e} c$ is (in the electron gas frame) root mean square transverse velocity. From (6) and (7) we would write rather, defining $V_{\perp}$ as root mean square transverse velocity,

$$
\begin{aligned}
k T /\left(m_{e} c^{2}\right) & =\left\langle V_{e^{-}}^{2}\right\rangle / c^{2}=\left\langle V_{e_{y}}^{2}\right\rangle / c^{2} \\
& =\frac{1}{2}\left\langle V_{e_{\perp}}^{2}\right\rangle / c^{2}=\frac{1}{2} V_{\perp}{ }^{2} / c^{2} .
\end{aligned}
$$

We will assume in what follows that this is is what they really had in mind, the authors of Refs. 2 and 3 , so that their definition of $\kappa$ is that of (8). This is suggested for Budker et al. by words after Eq. (11) of Ref. 1, (their root mean square is of just one of the two components of transverse velocity), and for Sørensen by what he quotes as his own result for $\kappa$, as we will see.

We find (in the non-magnetic case)

$$
\begin{array}{ll}
\text { Maxwell: } & \kappa=3 /(2 \sqrt{2 \pi})=0.60 \\
\text { flattened: } & \kappa=2 /(\pi \sqrt{2 \pi})=0.25
\end{array}
$$

In (11) we agree with Budker et al. ${ }^{1.2}$ but for the flattened case they have instead of (12)

$$
1 /(2 \pi)=0.16 \text {. }
$$

Comparison with Sørensen ${ }^{3}$ is not so immediate because he has a more sophisticated approach not assuming a constant Coulomb logarithm. His results, from numerical integration, emerge in numerical rather than analytical form; however, for beams with

$$
n_{e} \approx 3 \times 10^{8} \mathrm{~cm}^{-3}, \quad k T \approx 1 \mathrm{eV}
$$

he states that his results are fitted by (his equations $2.22,2.54$ )

$$
\begin{aligned}
\tau & =3 / I_{2} \\
I_{2} & =1.52 \times 10^{3} d r_{i} r_{e} n_{e} c\left(m_{e} c^{2} / k T\right)^{1.37} \gamma^{-2}
\end{aligned}
$$

corresponding to

$$
\begin{aligned}
\kappa & =\left(3 /\left(1.52 \times 10^{3}\right)\right)\left(m_{c} c^{2} / k T\right) \cdot{ }^{13} L_{c} \\
& =1.09 \times 10^{-2} L_{c} .
\end{aligned}
$$

This is close enough to Sørensen's own statement of accord with

$$
\begin{aligned}
\kappa & =(1 / 2 \pi) L_{c} / 15 \\
& =1.06 \times 10^{-2} L_{c}
\end{aligned}
$$

to confirm our reinterpretation above of his definition of $\kappa$. For the conditions (14) we find [from (25), (26) and (27) below]

$$
L_{c}=12.3,
$$

so that from (17)

$$
\kappa=0.13 .
$$

This is for the flattened distribution, and seems to agree more with Budker et al's (13) than with our (12). However, Sørensen considered only the damping of free motion. For oscillatory motion then his cooling time should be increased ${ }^{1,4}$ by a factor of 2 (see Section 3 below), already included in (11) and (12); then he agrees with us.

The numerical results (20) and (21) are for $k T$ $=1 \mathrm{eV}$. At first sight (16) might seem to require a $T$-dependent $\kappa$. But this would be to forget that $L_{c}$ is weakly $T$ dependent. The argument of the logarithm [(25), (26) and (27) below] goes as $T^{3 / 2}$. So we can generalize (20) to

$$
\begin{aligned}
L_{c}= & 12.3+\ln (k T / 1 \mathrm{eV})^{3 / 2} \\
& \propto 1+0.12 \ln (k T / 1 \mathrm{eV}) \approx(k T / 1 \mathrm{eV}) \cdot 12
\end{aligned}
$$

Allowing for this in (16) we see that Sørensen's results correspond to a rather constant $\kappa$.

In the following sections the arguments for (11) and (12) are set out in some detail, so that you can see if we go wrong. In Section 4 the magnetic case is taken up.

\section{NON-MAGNETIC CASE, NON- OSCILLATORY MOTION}

An ion penetrating an electron gas is supposed to be subject to a resisting force ${ }^{2}$ (in the rest frame of the gas)

$$
\begin{aligned}
\mathbf{F}\left(\mathbf{V}_{i}\right)= & K \int d^{3} \mathbf{V}_{e}\left(\mathbf{V}_{e}-\mathbf{V}_{i}\right) \\
& \times\left|\mathbf{V}_{e}-\mathbf{V}_{i}\right|^{-3} f\left(\mathbf{V}_{e}\right) c^{2}
\end{aligned}
$$

where

$$
\begin{gathered}
\mathbf{V}_{i} \text { is ion velocity } \\
K=4 \pi Z_{i}^{2} n_{e} r_{e}{ }^{3}\left(m_{e} c^{2} / r_{e}\right) L_{c}
\end{gathered}
$$

The Coulomb logarithm $L_{c}$ is more properly a function of $\mathbf{V}_{e}$ and to the right of the integral sign. 
But we suppose that it varies slowly and can be fixed at a typical value

$$
L_{c}=\ln \left(\rho_{\max } / \rho_{\min }\right)
$$

where, for small $V_{i}$

$$
\rho_{\min }=2 r_{e} c^{2} / V_{\perp}^{2}
$$

and (in an unbounded medium)

$$
\rho_{\max }=r_{c^{\prime}}\left(V_{\perp} / c\right) / \sqrt{4 \pi n_{c} r_{c^{\prime}}{ }^{3}},
$$

where $V_{\perp}$ is defined in (10).

The mathematical problem of calculating $\mathbf{F}$ for a given distribution $f\left(\mathbf{V}_{c}\right)$ is then the same $e^{4.5}$ as that of calculating the electrostatic field set up by a given charge distribution. Ideas familiar in electrostatics can be pressed into service, in particular Gauss' theorem relating the flux of field through a closed surface to the charge within:

$$
\int d \mathbf{s} \cdot \mathbf{F}(\mathbf{V})=-4 \pi \int d^{3} \mathbf{V} K f(\mathbf{V}) c^{2},
$$

where the first integral is over a closed surface in velocity space and the second over the volume contained by it.

\section{Maxwell distribution}

Consider first the symmetrical Maxwell distribution (3). By symmetry $\mathbf{F}(\mathbf{V})$ is in the direction V.

$$
\mathbf{F}=-\alpha\left(V^{2}\right) \mathbf{V}
$$

Applying (28) to a small sphere around the origin $4 \pi V^{2} \alpha(0) V$

$$
=4 \pi K(4 / 3) \pi V^{3}\left(2 \pi k T / m_{e}\right)^{-3 / 2} c^{2},
$$

whence

$$
\alpha(0)=(4 /(3 \sqrt{\pi}))\left(2 k T / m_{e}\right)^{-3 / 2} K c^{2} .
$$

So for small ion velocity (relative to the electron gas as a whole)

$$
\mathbf{F}\left(\mathbf{V}_{i}\right)=-(4 /(3 \sqrt{\pi}))\left(m_{e} c^{2} /(2 k T)\right)^{3 / 2} K \mathbf{V}_{i} / c
$$

If there are no other forces at work, (32) gives rise to exponential damping

$$
\mathbf{V}_{i} \propto \exp \left(-t / t_{0}\right)
$$

with mean life

$$
\begin{aligned}
t_{0} & =\left[3 \sqrt{\pi} m_{i} c /(4 K)\right]\left[2 k T /\left(m_{e} c^{2}\right)\right]^{3 / 2} \\
& =\frac{3}{16 \sqrt{\pi}}\left(\frac{2 k T}{m_{e} c^{2}}\right)^{3 / 2} \frac{m_{i}}{m_{e}} \frac{r_{e}}{c} \frac{1}{n_{e} r_{e}{ }^{3} L_{c}} .
\end{aligned}
$$

This agrees with Spitzer [Ref. 5, Eq. (5.31), with $\left.T_{i} / m_{i} \ll T_{c} / m_{e}\right]$ when it is remembered that he is interested in temperature, or energy, or velocity squared, rather than velocity, and so quotes a cooling time which is half of the above, because

$$
\left[\exp \left(-t / t_{0}\right)\right]^{2}=\exp \left[-t /\left(t_{0} / 2\right)\right]
$$

It also agrees with Budker et al. [Ref. 1, Eq. (11)] when it is noted that they have divided by a duty cycle factor ( $\eta$ in their notation, $d$ in ours) to allow for the application of cooling to only a fraction of the ion orbit, and multiplied by a factor of 2 as appropriate in application to betatron oscillation ${ }^{4}$ (see Section 3 ).

\section{Flattened distribution}

We are more interested in the "flattened" distribution (4). ${ }^{1.2}$ To calculate $\mathbf{F}(\mathbf{V})$ for small $V_{z}$, $V_{y}$ it suffices to calculate $F_{x}$ for $V_{z}=V_{y}=0$. For by applying Gauss' theorem to a small slim cylinder on the $V_{r}$ axis $(\mathrm{Cl}$, in the figure) of radius

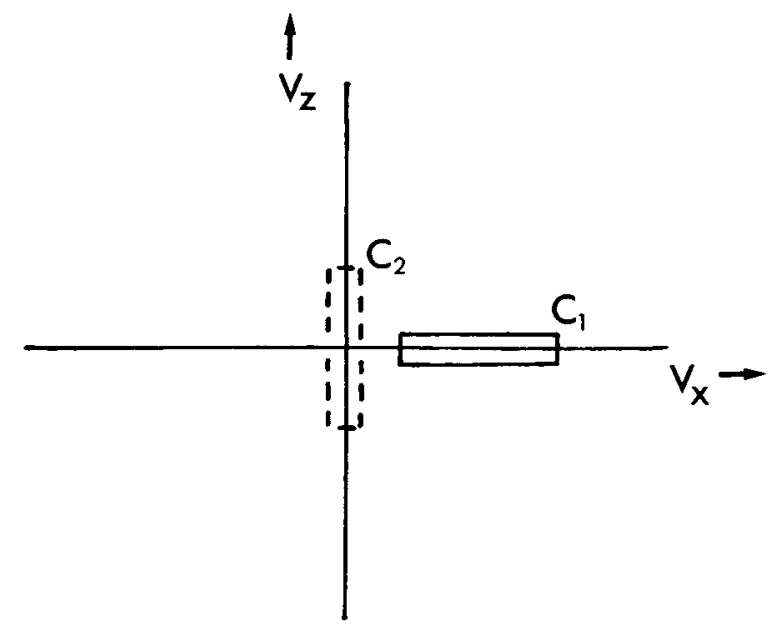

Small regions $C_{1}$ and $C_{2}$ in velocity space to which Gauss theorem is applied. They are cylinders symmetric about the $V_{x}$ axis. The flattened electron distribution lies in the $V_{x} V_{z}$ plane. 
$V$, and length $l$. We have

$$
\begin{aligned}
\pi V^{2} F_{x}(\epsilon+l) & -\pi V^{2} F_{x}(\epsilon) \\
& +2 \pi V F_{\perp} l=0
\end{aligned}
$$

that is,

$$
\pi V^{2} \partial F_{. x} / \partial V_{x}+2 \pi V F_{\perp}=0
$$

whence

$$
\mathbf{F}_{\perp}=-1 / 2 \mathbf{V}_{\perp}\left(\partial F_{x} / \partial V_{x}\right)
$$

for small $\mathbf{V}_{\perp}$, the derivative being evaluated on the axis $\mathbf{V}_{\perp}=0$.

As in electrostatics it is convenient to introduce a potential

$$
\begin{aligned}
F_{x}= & \left(\partial / \partial V_{x}\right) \Phi\left(V_{x}\right) \\
\Phi(x)= & c^{2} K \int_{0}^{\infty} 2 \pi r d r\left(x^{2}+r^{2}\right)^{-1 / 2} \\
& \times\left(2 \pi k T / m_{e}\right)^{-1} \exp \left[-1 / 2 m_{e} r^{2} /(k T)\right]
\end{aligned}
$$

Change the integration variable to

$$
R=\sqrt{\left(x^{2}+r^{2}\right)}
$$

Then

$$
\left.\begin{array}{l}
\Phi(x)=C \int_{x}^{\infty} d R \exp \left[-1 / 2 m_{e} R^{2} /(k T)\right] \\
C(x)=K\left[m_{e} c^{2} /(k T)\right] \exp \left[1 / 2 m_{e} x^{2} /(k T)\right]
\end{array}\right\}
$$

From (20), for $V_{x}$ positive,

$$
\begin{aligned}
F_{x}\left(V_{x}\right)= & -K\left[m_{e} c^{2} /(k T)\right] \\
+ & C\left(V_{x}\right)\left[m_{e} V_{x} /(k T)\right] \int_{V_{x}}^{\infty} d R \\
& \quad \times \exp \left[-1 / 2 m_{e} R^{2} /(k T)\right]
\end{aligned}
$$

(which can be expressed in terms of the tabulated error function erf). Then from (36) and (41) (following earlier papers, we write $\|$ for the $x$ direction, i.e., parallel to the cooler axis)

$$
\begin{aligned}
& F_{\|}=-\left(V_{i \|}|| V_{i \|} \mid\right) K m_{e} c^{2} /(k T) \\
& \mathbf{F}_{\perp}=-\mathbf{V}_{i \perp} \sqrt{\pi}\left[m_{e} c^{2} /(2 k T)\right]^{3 / 2} K / c
\end{aligned}
$$

for

$$
\left|\mathbf{V}_{i}\right| \ll \sqrt{\left(2 k T / m_{e}\right)}=V_{\perp} .
$$

Note that with (42), unlike (32), $F_{\|}$does not go to zero with $V_{i \|}$. The longitudinal cooling is then linear rather than exponential. However, ${ }^{3}$ with very small $V_{i \|}$ the theory used here fails (in particular the Coulomb logarithm does not remain constant), and $F_{\|}$does go finally to zero.

The transverse force (43) for the flattened distribution, as compared with the symmetrical case (32), for given $V_{i+}$ (i.e., given $T$ ), is bigger by a factor

$$
3 \pi / 4=2.36 .
$$

Note that (42) can be obtained more quickly by applying Gauss' theorem to a short fat cylinder on the axis (C2 dotted in the figure).

\section{BETATRON OSCILLATION DAMPING}

During a small time $\delta t$ the force (43) causes an increment in, say, the vertical ion velocity

$$
\delta V_{i z}=-V_{i z}(3 \pi / 4) \delta t / t_{0},
$$

with $t_{0}$ given by (33). In the absence of other forces, and with continuous application of the cooling, we would have exponential damping of $V_{i z}$ with mean life

$$
t_{0} 4 /(3 \pi) .
$$

But in the case of betatron oscillation there are other forces, causing $V_{i z}$ to oscillate.

We neglect here any coupling between horizontal and vertical betatron oscillation. Then the two degrees of freedom can be treated separately and similarly. At some particular position in the cooler let the undamped vertical velocity be

$$
V_{i z}=V_{i z \max } \sin (\mu n+\phi)
$$

on the $n$ 'th passage of the ion. The increment of velocity (46) adds an extra oscillation which, if induced on, say, the $o$ 'th passage goes subsequently as

$$
-V_{i z \max } \sin \phi(3 \pi / 4)\left(\delta t / t_{0}\right) \cos \mu n .
$$


Writing here

$$
\begin{aligned}
\cos \mu n= & \cos ((\mu n+\phi)-\phi) \\
= & \sin \phi \sin (\mu n+\phi) \\
& +\cos \phi \cos (\mu n+\phi)
\end{aligned}
$$

we have for the combined oscillation $(48)+(49)$

$$
\begin{aligned}
& \sin (\mu n+\phi)\left\{V_{i z \max }\right. \\
& \left.\quad-V_{i z \max } \sin \phi \sin \phi(3 \pi / 4) \delta t / t_{0}\right\} \\
& +\cos (\mu n+\phi)\left\{-V_{i z \max } \sin \phi \cos \phi(3 \pi / 4) \delta t / t_{0}\right\}
\end{aligned}
$$

or

$$
\left(V_{i z \max }+\delta V_{i z \max }\right) \sin (\mu n+\phi+\delta \phi)
$$

with, to first order in $\delta t$,

$$
\delta V_{i z \max }=-V_{i z \max }(\sin \phi)^{2}(3 \pi / 4) \delta t / t_{0} .
$$

We suppose that the damping becomes appreciable only after many passages of the ion through the cooler. Then in (51) we may average over all values of the phase $\phi$

$$
(\sin \phi)^{2} \rightarrow 1 / 2 .
$$

If we also take account of the fact that damping occurs only in the cooler, and so only for a fraction

$$
d=\text { (cooler length)/(orbit length) }
$$

of the time, we have finally on average

$$
\left(-d V_{i z \max } / d t\right) / V_{i z \max }=3 \pi d /\left(8 t_{0}\right) .
$$

This implies exponential damping

$$
V_{i z \max } \propto \exp \left(-t / t_{\beta}\right)
$$

with mean life

$$
t_{\beta}=t_{0} 8 /(3 \pi d) .
$$

Apart from the trivial duty cycle factor, (56) has doubled as compared with (47) because of the oscillatory nature of the undamped motion. Casting (56) into the form (8), and noting that $\gamma^{2}$ is a relativistic correction arising in transformation between lab. and electron gas systems, gives (12). Taking out the flattening effect (45) gives (11).

\section{MAGNETIC CASE}

Suppose now that a strong magnetic field is applied in the longitudinal direction. ${ }^{7}$ The ion-electron encounters are conventionally divided, crudely, into two kinds, "fast" and "adiabatic." The "fast" contribution to the drag force is again (42) and (43), except that the Coulomb logarithm in $K$ is replaced

$$
\begin{aligned}
& L_{c} \rightarrow L_{f} \\
& L_{f}=\ln \left(r_{L} / \rho_{\min }\right),
\end{aligned}
$$

where $r_{L}$ is a typical electron Larmor radius

$$
r_{L}=m_{e} c\left(2 k T / m_{e}\right)^{1 / 2} /(e B) \text {. }
$$

The "adiabatic" contribution ${ }^{7-9}$ is rather complicated and couples together in general the three degrees of freedom. We will consider only particular simple cases.

$V_{i \perp}=O$ (i.e., no betatron oscillation)

In this case there is no "adiabatic" contribution, and the complete drag force is (42) with (58)

$$
\begin{aligned}
F_{\|}= & -\left(V_{i \|} /\left|V_{i||}\right|\right)\left(m_{e} c^{2} / k T\right) \\
& \times\left(4 \pi Z_{i}{ }^{2} n_{e} r_{e}{ }^{3} L_{f}\right)\left(m_{e} c^{2} / r_{e}\right)
\end{aligned}
$$

$$
\begin{aligned}
& V_{i \|}=V_{i y}=O \text { (i.e., pure vertical betatron } \\
& \text { oscillation) }
\end{aligned}
$$

Horizontal betatron oscillation is entirely similar. Note, however, that we omit here any coupling between horizontal and vertical betatron oscillation, including that due to the cooler magnetic field.

The complete drag force is now

$$
\begin{aligned}
F_{z}\left(V_{i z}\right)= & -V_{i z}\left(K / L_{c}\right)\left\{\sqrt{\pi}\left(2 k T / m_{\iota}\right)^{-3 / 2} L_{f}\right. \\
& \left.+1 / 2\left|V_{i z}\right|^{-3} L_{a r}\right\} c^{2},
\end{aligned}
$$

where the two terms are "fast" and "adiabatic" contributions respectively; $L_{f}$ is again (58) and

$$
L_{a}=\ln \left(\rho_{a \max } / r_{L}\right),
$$

where $r_{L}$ is again (59) and

$$
\left.\rho_{a \max }=r_{e}\left(\left|V_{i z}\right| / c\right) / \sqrt{\left(4 \pi n_{e} r_{c}{ }^{3}\right.}\right) .
$$


In a small slice of cooler we now have

$$
\delta V_{i z}=F_{z}\left(V_{i z}\right) \delta t / m_{i}
$$

giving rise to an increase in oscillation amplitude [for $n=0$ in (48)]

$$
\delta V_{i z \max }=\left(F_{z}\left(V_{i z}\right) / m_{i}\right) \delta t \sin \phi .
$$

Averaging over $\phi$ and around the orbit

$$
\begin{aligned}
& m_{i}\left(d V_{i z \max } / d t\right) \\
& \quad=(2 d / \pi) \int_{0}^{\pi / 2} d \phi \sin \phi F_{z}\left(V_{i z \max } \sin \phi\right)
\end{aligned}
$$

In (66) the second term of (61) gives rise to the divergent integral

$$
\int_{0}^{\pi / 2} d \phi /(\sin \phi)
$$

In this connection we have to recognize that the theory predicting unlimited rise of $F_{z}$ with decrease of $V_{z}$ must fail for sufficiently small $V_{z}$. It will no longer be permissible to neglect the spread of longitudinal electron velocity, or motion in the other transverse degree of freedom, or the variation of the logarithm $L_{a}$, or whatever. Then the divergent integral is cut off at some velocity

$$
V_{i z \text { min }}
$$

or corresponding phase

$$
\sin \phi_{\min }=V_{i z \min } / V_{i z \max } .
$$

Note that

$$
\begin{aligned}
\int_{\phi_{\text {min }}}^{\pi / 2} d \phi /(\sin \phi) & =-\ln \tan \left(\phi_{\min } / 2\right) \\
& \approx \ln \tan \left(2 V_{i z \max } / V_{i z \min }\right) .
\end{aligned}
$$

Then (66), with the explicit form (61) for $F_{z}$, becomes

$$
\begin{aligned}
(- & \left.d V_{i z \max } / d t\right) / V_{i z \max } \\
& =c^{2} K d /\left(L_{c} m_{i}\right)\left\{\sqrt{\pi}\left(2 k T / m_{\ell}\right)^{-3 / 2} L_{f} / 2\right. \\
& +1 / 2\left(V_{i z \max }\right)^{-3} L_{a}(2 / \pi) \\
& \left.\times \ln \left(2 V_{i z \max } / V_{i z \min }\right)\right\}
\end{aligned}
$$

or

$$
\left(-d V_{i z \max } / d t\right) / V_{i z \max }=t_{f}^{-1}+t_{a}^{-1},
$$

with, in terms of $t_{0}$ defined in (33),

$$
\begin{aligned}
t_{f}= & t_{0}[8 /(3 \pi d)] L_{c} / L_{f} \\
t_{a}= & t_{f}\left(V_{i z \max }\right)^{3}\left(2 k T / m_{e}\right)^{-3 / 2} \\
& \times\left(L_{f} / L_{a}\right) \sqrt{\pi} / R \\
R= & (2 / \pi) \ln \left(2 V_{i z \max } / V_{i z \min }\right) .
\end{aligned}
$$

The determination of $V_{i z \min }$ in (76) depends on particular study of particular cases. So the application of the result is not immediate. It perhaps serves, however, to indicate the complexity of the problem. It should be borne in mind moreover that only simplified cases have been considered here. The general problem is one of non-linear equations and coupled motion in three degrees of freedom, and with an electron distribution varying radially. Then, it seems to us, computer simulation is indicated, and a promising start on that has been made. ${ }^{10}$

\section{ACKNOWLEDGEMENTS}

We warmly thank Allan Hvidkjaer Sørensen for correspondence confirming our interpretation of his work, and indeed for showing us a manuscript in which he explicitly and independently derives the factor $3 \pi / 4$ for the flattening effect, and Frank Krienen for several useful remarks.

\section{REFERENCES}

1. G. I. Budker et al., Particle Accelerators 7197 (1976).

2. Ya. S. Derbenev and I. Meshkov, CERN yellow report, CERN 77-08 (1977).

3. A. H. Sørensen, preliminary internal report 1, Aarhus (1978).

4. Ya. S. Derbenev and A. N. Skrinsky, Particle Accelerators 81 (1977).

5. F. Mills, Fermilab unpublished (1976).

6. L. Spitzer, Physics of Fully Ionized Gases (Wiley, New York, 1962).

7. Ya. S. Derbenev and A. N. Skrinsky, Particle Accelerators 8235 (1978).

8. A. H. Sørensen, preliminary internal report 2, Aarhus (1978).

9. M. Bell, Particle Accelerators 10101 (1980).

10. M. Bell, CERN-EP Internal Report 79-10, December 1979. 